\title{
Connection between the period and the amplitude of the Blazhko effect
}

\author{
Benkő, József M.a and Szabó, Róbert \\ Konkoly Observatory, MTA CSFK, Konkoly Thege u. 15-17. H-1121 Budapest, Hungary
}

\begin{abstract}
We found a possible relationship between the modulation period and the amplitude of the Blazhko RR Lyrae stars: long modulation period generally implies high modulation amplitude while the short modulation period results in small amplitude. Although this effect is much more a tendency than a strict rule, it can be detected easily in the space-born time series data produced by Kepler and CoRoT. Good quality ground-based data show this relation, too. This phenomenon could give us constraints for the physics of the Blazhko effect.
\end{abstract}

\section{Introduction}

By investigating the Kepler Blazhko RR Lyrae light curves [3] we found a possible connection between the Blazhko period $P_{\mathrm{B}}$ and the Fourier amplitude of the modulation frequency $A\left(f_{\mathrm{B}}\right)\left(P_{\mathrm{B}} \propto A\left(f_{\mathrm{B}}\right)\right.$; see Fig. 9 in [3]). The literature was reviewed searching for this effect, but nothing specific was found. Two papers were published which implicitly suggest such a relation. (i) Jurcsik, Sódor \& Váradi [6] found a correlation between the pulsation period $P_{0}$ and the modulation amplitude of RR Lyrae stars $\left(P_{0} \propto A_{2}\right)$. Here the modulation amplitude $A_{2}$ was defined as the sum of the Fourier amplitudes of the first four modulation components: $A_{2}=A\left(f_{0}+f_{\mathrm{B}}\right)+A\left(f_{0}-f_{\mathrm{B}}\right)+A\left(2 f_{0}+f_{\mathrm{B}}\right)+A\left(2 f_{0}-f_{\mathrm{B}}\right)$. (ii) Jurcsik et al. [7] found a correlation between $P_{0}$ and the Blazhko period $P_{\mathrm{B}}$, as well $\left(P_{0} \propto P_{\mathrm{B}}\right)$.

On the basis of these works we could deduce a possible relation between the Blazhko period and the modulation strength: viz. if $P_{0} \propto A_{2}$ and $P_{0} \propto P_{\mathrm{B}}$ then $P_{\mathrm{B}} \propto A_{2}$. What is the connection between the parameter $A_{2}$ and $A\left(f_{\mathrm{B}}\right)$ ? As we showed in a simplified mathematical framework [2], the modulation component amplitudes in $A_{2}$ depend on the strength of the frequency modulation (FM), while $A\left(f_{\mathrm{B}}\right)$ depends on the amplitude modulation (AM) only. Therefore, using $A\left(f_{\mathrm{B}}\right)$ is clearly superior in characterizing AM. There is, however, a drawback. The number of stars of known $A\left(f_{\mathrm{B}}\right)$ is small, because $f_{\mathrm{B}}$ can be detected only in space-born and the best quality ground-based data.

\section{Sample and Method}

We collected stars where $A\left(f_{\mathrm{B}}\right)$ are known. These stars were observed from space by Kepler [3], by CoRoT [4], [11], [5], and from the ground by the Konkoly Blazhko Survey (KBS, [8]). These different observations were obtained in different color bands (e.g. $V, R, I, K_{\mathrm{p}}$ ), hampering their uniform handling. The spectral response function of the CoRoT and Kepler detectors are similar, but the CoRoT band is a bit wider than Kepler's one [1], [12], therefore, we have to scale the CoRoT amplitudes. Nemec at al. [10] found empirical transformations between amplitudes (e.g. $A_{\mathrm{tot}}, A_{1}$ ) in bands $K_{\mathrm{p}}$ and $V$. The transformed KBS data, however, do not show a clear correlation. There is an alternate parameter for characterizing the strength of AM: the amplitude of the envelope curve $A_{\max }$. This parameter can be determined easily, but it depends also on the pulsation amplitude. We checked the ratio on KBS data

\footnotetext{
a e-mail: benko@konkoly.hu
}

This is an Open Access article distributed under the terms of the Creative Commons Attribution License 4.0, which permits unrestricted use, distribution, and reproduction in any medium, provided the original work is properly cited. 


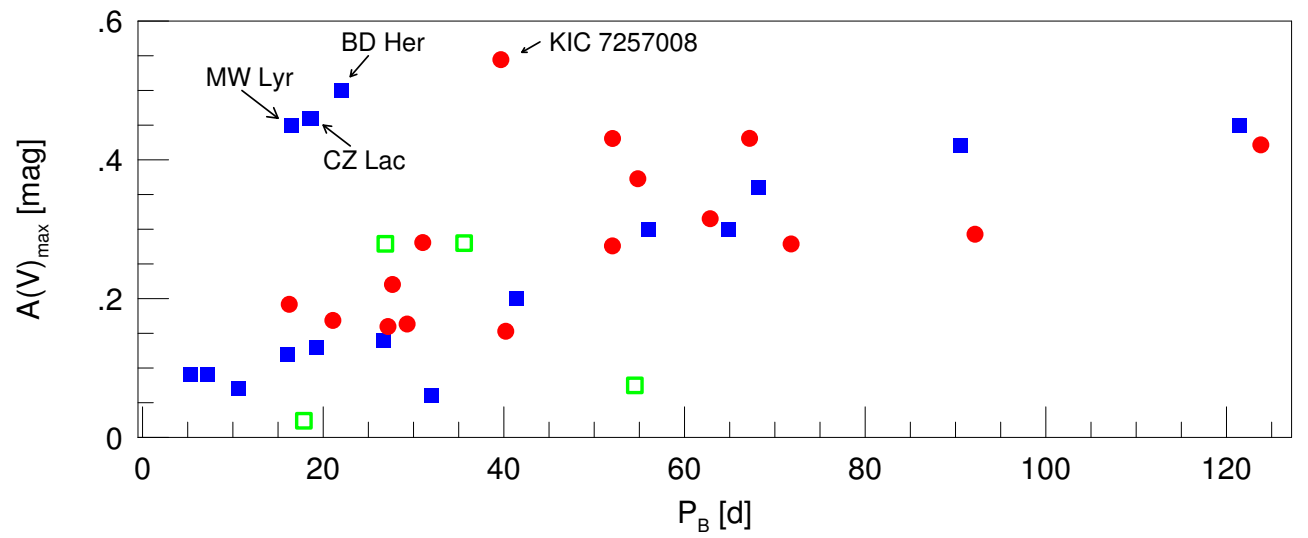

Fig. 1. Blazhko period $P_{\mathrm{B}}$ vs. total $V$ amplitude of the maxima (envelope) curve. Red and blue symbols denote Kepler and Galactic field (Konkoly Blazhko Survey) RR Lyrae stars, respectively. Open (green) squares indicate transformed values of the CoRoT stars. The typical errors are smaller than the symbols.

using this $A_{\max }$ values and found a tight correlation between $P_{\mathrm{B}}$ and $A(V)_{\max }$ (see blue symbols in Fig. 1). We applied the empirical transformation formula of [10] between the total amplitude in bands $K_{\mathrm{p}}$ and $V$. If we plot the result in Fig. 1. (red circles), Kepler stars show similar correlation than KBS stars. This $P_{\mathrm{B}} \propto A_{\max }$ ratio could be tested in the future by using massive photometric data basis (e.g. MACHO, OGLE).

\section{Interpretation}

Similar effect is common in hydrodynamical systems: e.g. weakly dissipating systems forced to show high amplitude by long time-scale perturbing forces only [9]. We call the reader's attention to the deviating stars (CZ Lac, MW Lyr, BD Her and KIC 7257008 in Fig. 1 and V355 Lyr in Fig. 9 of [3]). These may represent a separate group within Blazhko RR Lyrae stars. The existence of this group can also be tested by using large data bases.

The research leading to these results has received funding from the European Community's Seventh Framework Programme (FP7/2007-2013) under grant agreements no. 269194 (IRSES/ASK) and no. 312844 (SPACEINN). This work was also supported by the following grants: ESA PECS No 4000103541/11/NL/KML, No 4000110889/14/NL/NDe and the Hungarian OTKA Grant K-83790.

\section{References}

1. Auvergne, M. et al. A\&A 506 (2009) A411

2. Benkő, J.M., Szabó R. \& Paparó, M. MNRAS 417 (2011) 974

3. Benkó, J.M. et al. ApJS 213 (2014) 31

4. Chadid, M. et al. A\&A 510 (2009) A39

5. Guggenberger, E. et al. MNRAS 415 (2011) 1577

6. Jurcsik, J., Sódor Á. \& Váradi M. IBVS (2005) No 5666

7. Jurcsik, J. et al. AcA 55 (2005) 303

8. Jurcsik, J. et al, MNRAS 400 (2009) 1006

9. Molnár, L., Kolláth, Z. \& Szabó, R. MNRAS 424 (2012) 31

10. Nemec, J.M. et al. MNRAS 417 (2011) 1022

11. Poretti, E. et al. A\&A 520 (2010) A108

12. Van Cleve, J. et al. The Kepler Instrumental Handbook (NASA Ames, 2009) pp 103 